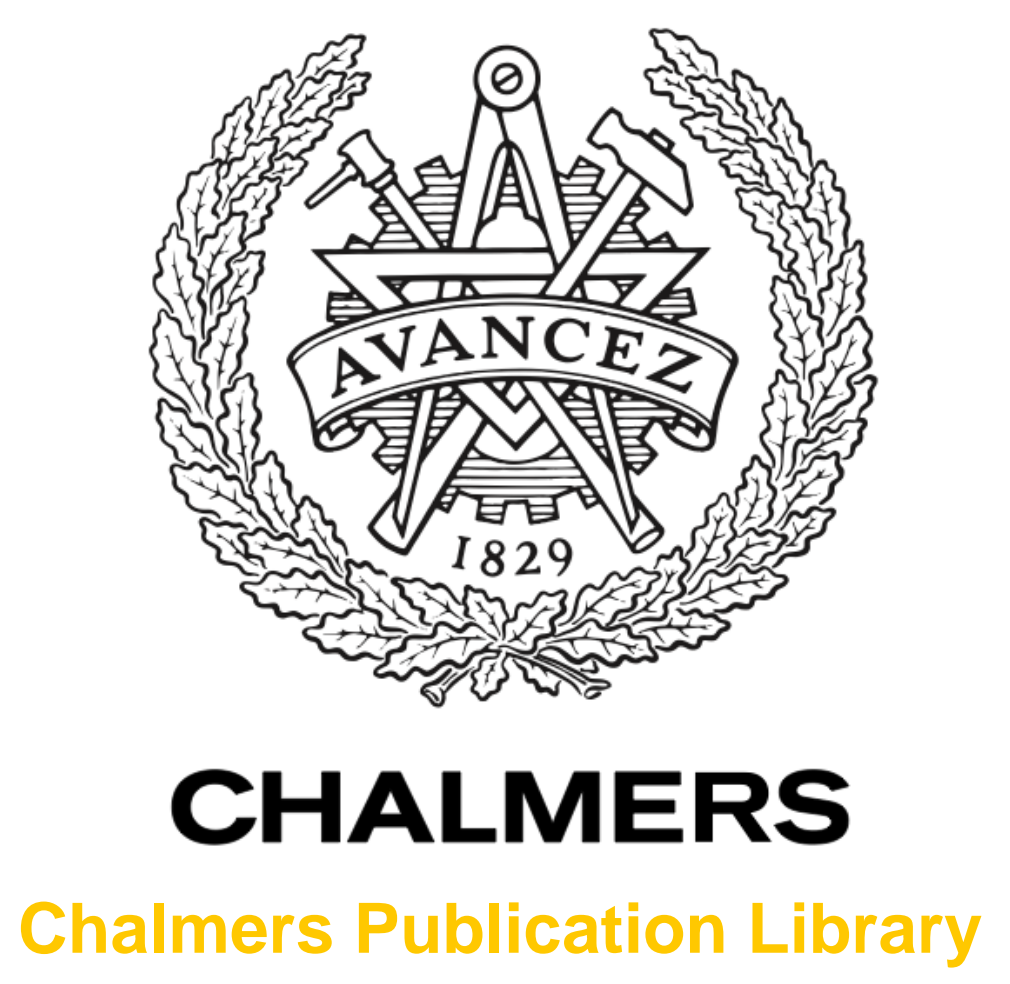

\title{
Matrix receiving scheme supporting arbitrary multiple-wavelength reception for optical interconnects
}

This document has been downloaded from Chalmers Publication Library (CPL). It is the author's version of a work that was accepted for publication in:

\section{European Conference on Optical Communication (ECOC)}

Citation for the published paper:

Lu, Y. ; Agrell, E. ; Pang, X. et al. (2017) "Matrix receiving scheme supporting arbitrary multiple-wavelength reception for optical interconnects". European Conference on Optical Communication (ECOC)

Downloaded from: http://publications.lib.chalmers.se/publication/254767

Notice: Changes introduced as a result of publishing processes such as copy-editing and formatting may not be reflected in this document. For a definitive version of this work, please refer to the published source. Please note that access to the published version might require a subscription. 


\title{
Matrix Receiving Scheme Supporting Arbitrary Multiple- Wavelength Reception for Optical Interconnects
}

\author{
Yang Lu ${ }^{1,4}$, Erik Agrell ${ }^{2}$, Xiaodan Pang ${ }^{1}$, Oskars Ozolins ${ }^{3}$, Xuezhi Hong ${ }^{1}$, Rui Lin ${ }^{1}$, Yuxin Cheng ${ }^{1}$, \\ Aleksejs Udalcovs ${ }^{3}$, Sergei Poppv ${ }^{1}$, Gunnar Jacobsen ${ }^{3}$ and Jiajia Chen ${ }^{1}$ \\ (1) School of ICT, KTH Royal Institute of Technology, Kista, Sweden, (luyang@hdu.edu.cn) \\ (2) Department of Electrical Engineering, Chalmers University of Technology, Göteborg, Sweden \\ (3) Networking and Transmission Laboratory, RISE Acreo AB, Kista, Sweden \\ (4) College of Communication Engineering, Hangzhou Dianzi University, Hangzhou, China
}

\begin{abstract}
An arbitrary multiple-wavelength reception scheme using only a few fixed-wavelength filters is proposed for optical interconnects. Filter matrices design based on error-control coding theory is devised. The feasibility of the proposed scheme is demonstrated in a four-wavelength reception experiment.
\end{abstract}

\section{Introduction}

rapidly increasing of internet traffic drives the need for high-speed and large-scale data center networks (DCNs). Optical interconnects have been introduced to sustain the future traffic demand in DCNs ${ }^{1,2}$. Particularly, passive optical interconnects (POls), which only use passive optical components for interconnection of the servers within the data centers ${ }^{3-6}$, are considered as a promising solution to provide high energy efficiency. It has been demonstrated that the power consumption can be reduced by a factor of 10 using POls instead of commodity switches ${ }^{3}$. Besides, thanks to passive optical components, POls also have advantages in terms of low cost, high reliability, easy maintenance and transparency to the data rate and modulation format.

There are two major types of POls, namely the coupler-based ones,4 and the arrayed waveguide grating (AWG)-based ones ${ }^{6,7}$. The coupler-based POls suffer from high insertion loss and have difficulties to support large-scale optical interconnects ${ }^{8}$. High insertion loss can be avoided in the AWG-based POIs. In principle, the insertion loss of an $N \times N$ cyclic AWG is not affected by the size of the POIs and can be kept relatively low.

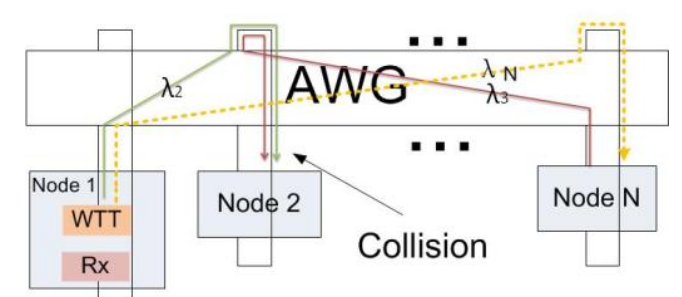

Fig. 1: A collision in an AWG based POI.

A typical AWG-based POI is shown in Fig. 1. Each node is equipped with a wavelengthtunable transmitter (WTT). By adjusting the transmitted wavelength, each node could send data to any other node. For example, Node 1 sends data to Node 2 by $\lambda_{2}$. If Node 1 sends data to Node $N$, the wavelength should be tuned to $\lambda_{N}$. As the routing is achieved by using the WTT and the AWG, the receiver (Rx) does not necessarily have to be wavelength-selective, which further reduces the complexity of the AWG-based POls compared to the couplerbased ones.

However, in such an AWG-based POI, there is a collision if several nodes send data to the same destination, as shown in Fig. 1. To avoid the collisions, one solution is to make each node capable of simultaneously receiving multiple wavelengths. As the incoming signals may be from any other nodes (in other words, carried by any wavelengths), signal separation becomes a challenge. The direct way is to use multiple wavelength-tunable filters. However, due to the immature technique, tunable filters are still expensive and with limited capability to achieve high tuning speed and large tuning range simultaneously. Without any wavelength-tunable components, an alternative solution is to equip each node with an large array of fixedwavelength filters and Rxs that match the total number of wavelengths. However, it is costly and the utilization of the Rxs may be low, especially for large-scale POls.

In this paper, we propose a matrix receiving scheme, which allows for receiving the signals carried by arbitrary multiple-wavelength simultaneously using only a limited number wavelength fixed filters and Rxs. Each filter covers several wavelengths and there can be overlaps between their pass bands. As a result, each $\mathrm{Rx}$ receives the signal carried by one wavelength or a combination of several wavelengths. With a proper configuration of the filter matrix, the signals, no matter by which wavelengths they are carried, can be recovered.

\section{Matrix receiving scheme}

An example of the proposed matrix receiving scheme for an AWG-based POI using in total 15 wavelengths is illustrated in Fig. 2. Each node is 
equipped with four filters and Rxs. The filters' pass bands can be depicted by a filter matrix (see Fig. 2). Each row of the matrix corresponds to a filter and each column corresponds to a wavelength. The element " 1 " or "0" means that the wavelength can or cannot pass the corresponding filter.

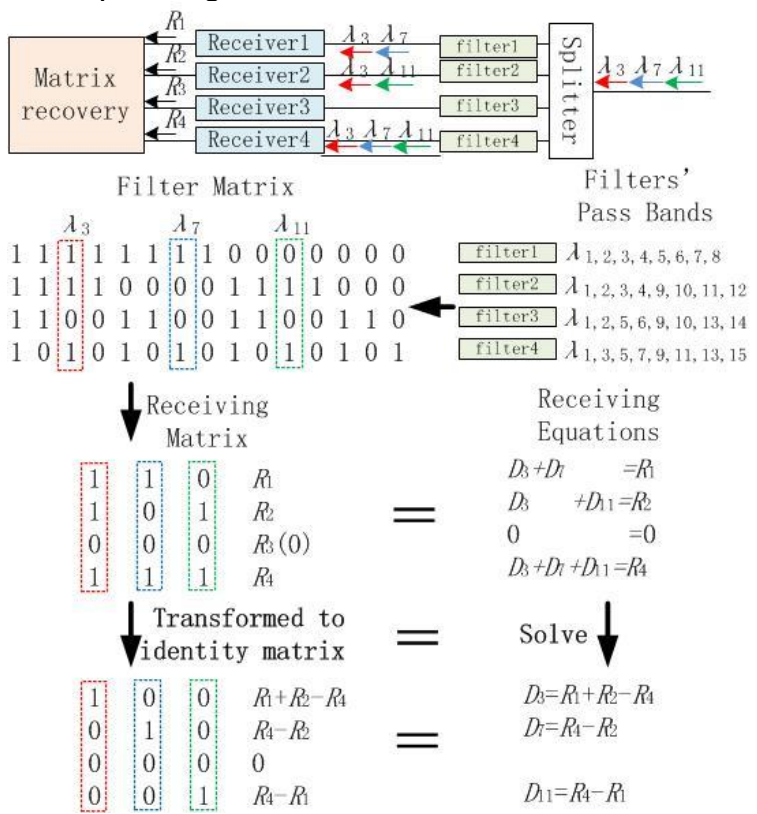

Fig. 2: A matrix receiving scheme with four filters and Rxs for an AWG-based POI using 15 wavelengths.

For the example shown in Fig. 2, three signals $D_{1}, D_{2}$, and $D_{3}$ carried by $\lambda_{3}, \lambda_{7}$, and $\lambda_{11}$ arrive at the same time. The signals should be synchronized at the bit level and of the same power. Each Rx outputs its own combination of input signals, here called $R_{1}, R_{2}, R_{3}$, and $R_{4}$. Columns 3,7 , and 11 of the filter matrix compose a receiving matrix, which corresponds to the receiving equations.

I If the columns in the receiving matrix are linearly independent, the receiving equations are solvable. We can get the solutions by transforming the receiving matrix to an identity one and then we can recover the signals carried by each wavelength from the filter outputs.

\section{Filter matrix design}

The filter matrix shown in Fig. 2 is not universal. In fact, it is designed to simultaneously receive signals carried by up to two wavelengths. If more wavelengths arrive at the same time, in some cases the receiving equations become unsolvable. For example, columns 4, 8, and 12 in the filter matrix are linearly dependent, so we cannot recover the signals if they are carried by $\lambda_{4}, \lambda_{8}$, and $\lambda_{12}$. In general, a filter matrix should be designed to maximize the number of wavelengths that can be simultaneously received, $M$, for a given total number of wavelengths, $N$, and a given number of filters and Rxs, $F$. With this notation, a filter matrix is an $F \times N$ binary matrix, in which any $M$ columns are linearly independent.

One solution to the filter matrix design is using error-control coding theory. A binary block code with parameters $(n, k, d)$ is a set of $2^{k}$ binary codewords with length of $n$, such that every pair of distinct codewords differ in at least $d$ positions ${ }^{9}$. If the code is linear, it is fully specified by a binary parity-check matrix $\mathbf{H}$ with dimensions $(n-k) \times n$. Any $d-1$ columns in $\mathbf{H}$ are linearly independent. Therefore, any paritycheck matrix $\mathbf{H}$ for a binary block code with parameters $(n, k, d)$ can be applied as a filter matrix with parameters $N=n, F=n-k$, and $M=$ $d-1$. A rich literature exists on binary block code design and extensive tables are available giving the optimum number of codewords of a binary block code for given $n$ and $d^{10,11}$.

The arithmetics in coding theory is carried out in the binary Galois field GF(2). Linear independence in $G F(2)$ is a sufficient but not necessary condition for linear independence in the real-number field. Thus, coding theory provides one solution for the filter matrix design, but not always the optimal one.

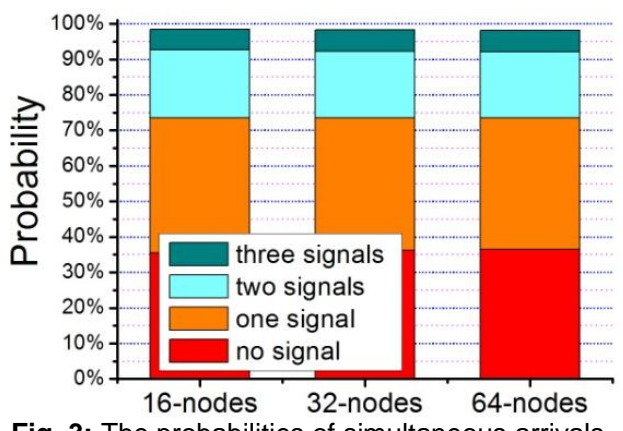

Fig. 3: The probabilities of simultaneous arrivals.

In fact, $M$ is often small. Assuming as a worst case that all nodes transmit in all time slots, and that the receiving node is randomly chosen with probability $1 /(N-1)$, the probabilities of simultaneous arrivals for the signals carried by different wavelengths are shown in Fig. 3 . The probability for signals carried by more than 3 wavelengths to arrive at the same destination simultaneously is rather low, regardless of the total number of nodes $N$ in the POI. Thus, designing filter matrices with $M=2$ and $M=3$ is important.

For $M=2$, binary block codes with parameters $(n, k, 3)$ already provide optimal filter matrices. Such codes are known to be (possibly shortened) Hamming codes, for which $k=n-$ $\left\lceil\log _{2}(n+1)\right]$. Thus, $F$ filters are able to support $\left[2^{F}-1\right]$ wavelengths. For $M=3$, improved filter matrices can be composed by the columns in 
which the number of " 1 " elements is from $p$ to $2 p-1$, for any constant $p$. Any three such columns are linearly independent, because the sum of any two columns has no less than $2 p$ " 1 " elements, impossible to match in the third one. For the columns of length $F$, there are $\sum_{i=p}^{2 p-1}\left(\begin{array}{l}F \\ p\end{array}\right)$ available candidates. To get most available candidates, i.e., the largest $N$, the optimum $p$ should be $\lceil(F+1) / 3]$. The relations between $F$ and $N$ are shown in Fig. 4. The required number of filters and Rxs, $F$ is still much lower than the number of total wavelengths, $N$.

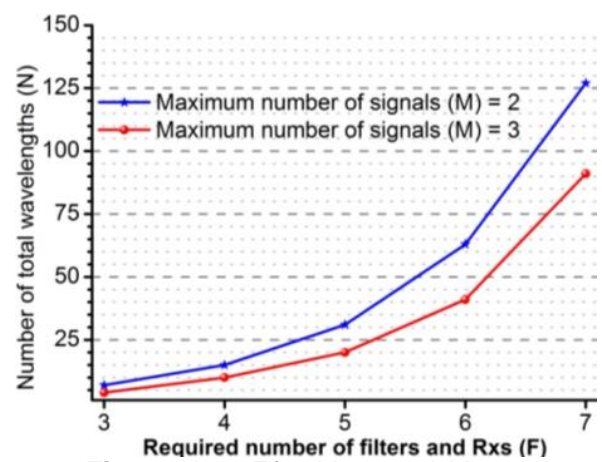

Fig. 4: $N$ vs. $F$ for $M=2$ and $M=3$.

\section{Experimental validation}

To verify the capability of multi-wavelength reception offered by the proposed matrix receiving scheme, we carry out a proof-ofconcept experiment.

We generated four $10 \mathrm{~Gb} / \mathrm{s}$ on-off-keying (OOK) modulated optical channels at $1530 \mathrm{~nm}$, $1550 \mathrm{~nm}, 1555 \mathrm{~nm}$ and $1556 \mathrm{~nm}$, repectively. The wavlengths are chosen purposely to cover both wide and narrow channel spacing cases. After bit synchronization and power adjustment, the signals were combined and detected by a photo detector. (a)

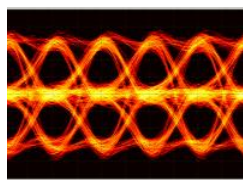

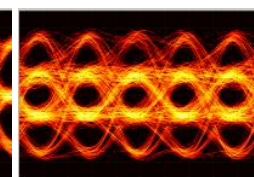

(b)

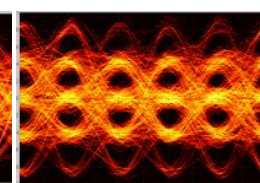

(c)
Fig. 5: Eye diagrams of received signal combining (a) 2, (b) 3 , and (c) 4 wavelengths.

The eye diagrams of the signals carried by different numbers of wavelengths that arrive simultaneously are measured at different received power and shown in Fig. 5. We tested the $Q$ factors of the eye diagrams and estimated the sensitivities (@FEC limit of BER=3.8E-3) at reception. The receiving sensitivities were -22.3 , -19.8 , and $-16 \mathrm{dBm}$ for the signals carried by 2 , 3 , and 4 wavelengths, respectively. Considering that the measured received power is the sum of all signals' powers, the nomalized receiving sensitivity is $-25.3,-24.8$, and $-22 \mathrm{dBm}$, respectively. The total link loss consists of an AWG loss (typically $5 \mathrm{~dB}$ ), a power splitter loss $\left(10 \log _{10} F \mathrm{~dB}\right.$, typically $6-15 \mathrm{~dB}$ ) and a filter loss (typically $1 \mathrm{~dB}$ ). With such assumption of link loss, the required transmitting power can be kept below $0 \mathrm{dBm}$, which implies tha commercial available transceivers can be employed in the proposed scheme.

\section{Conclusions}

To avoid collisions, a matrix receiving scheme is proposed that allows for receiving multiple wavelengths simultaneously. By using a few fixed wavelength filters, the proposed matrix receiving scheme is able to recover all the signals no matter by which wavelength they are carried. The filter matrix design was achieved using tools from error-control coding theory. An experimental demonstration verifies the capability of multi-wavelength reception that can be realized by commercially available transceivers.

\section{Acknowledgements}

This work was supported by the Swedish Research Council, Swedish Foundation for Strategic Research, Göran Gustafssons Stiftelse, Natural Science Foundation of Guangdong Province (Grant No. 508206351021) and National Natural Science Foundation of China (Grant No. 61550110240, $61671212)$. The equipment was funded by Knut and Alice Wallenberg foundation.

\section{References}

[1] C. Kachris et al., "A survey on optical interconnects for data centers," IEEE Communications Surveys \& Tutorials, Vol. 14, no. 4, pp. 1021-1036 (2012).

[2] C. Kachris et al., "Optical interconnection networks in data centers: Recent trends and future challenges," IEEE Commun. Mag., Vol. 51, no. 9, pp. 39-45 (2013).

[3] M. Fiorani et al., "Energy-efficient elastic optical interconnect architecture for data centers," IEEE Commun. Lett., Vol. 18, no. 9, pp. 1531-1534 (2014).

[4] Y. Gong et al., "Passive optical interconnects at top of the rack: Offering high energy efficiency for datacenters," Opt. Express, Vol. 23, no. 6, pp. 7957-7970 (2015).

[5] Y. Cheng et al., "Reliable and cost efficient passive optical interconnects for data centers," IEEE Commun. Lett., Vol. 19, no. 11, pp. 1913-1916 (2015).

[6] X. Hong et al., "AWG based passive optical interconnects for datacenters," in Proc. Photonic Networks and Devices, NeTu1C.3, Vancouver, Canada ( 2016).

[7] T. Ye et al., "Modular AWG-based interconnection for large-scale data center networks," IEEE Trans. Cloud Comput., to be published (2017).

[8] R. Lin et al., "Experimental Validation of Scalability Improvement for Passive Optical Interconnect by Implementing Digital Equalization", Proc. ECOC, Dusseldorf (2016).

[9] G. C. Clark et al., "Error-Correction Coding for Digital Communications". New York: Springer, 1981.

[10] E. Agrell, "Tables of binary block codes," online: http://codes.se/ bounds.

[11] E. Agrell et al., "A table of upper bounds for binary codes," IEEE Transactions on Information Theory, Vol. 47, no. 7, pp. 3004-3006(2001). 